\title{
Systemic administration of human umbilical cord-derived mesenchymal stem cells effectively ameliorates the outcomes of a critically ill elderly patient with COVID-19 with multiple comorbidities: A case report
}

\author{
HONGJUN CHEN $^{1 *}$, LEISHENG ZHANG ${ }^{2-4 *}$, ZHIXU HE $^{5^{*}}$, DALI WANG $^{6}$, LILI LIU $^{7}$, \\ WEI ZHANG ${ }^{1}$, TAO CHEN ${ }^{1}$, ZHIHUA DAI ${ }^{8}$, ZHONGCHAO HAN $^{2,3}$ and MIAO CHEN ${ }^{1}$

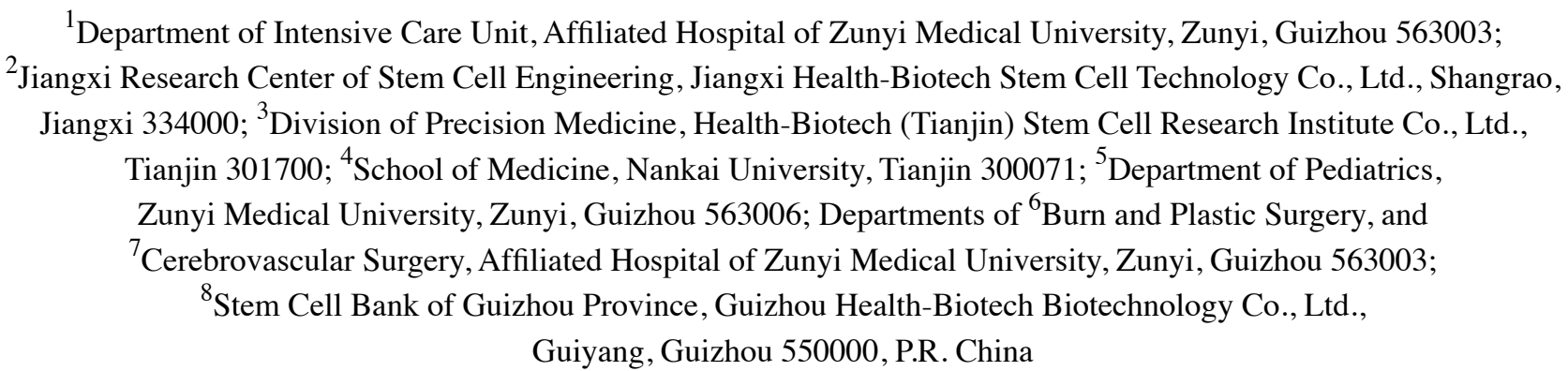

Received July 22, 2020; Accepted September 28, 2020

DOI: $10.3892 /$ wasj.2020.70

Abstract. The outbreak of coronavirus disease 2019 (COVID-19) caused by severe acute respiratory syndrome

Correspondence to: Professor Zhongchao Han, Jiangxi Research Center of Stem Cell Engineering, Jiangxi Health-Biotech Stem Cell Technology Co., Ltd., 001 Lanjiang Road, Shangrao, Jiangxi 334000, P.R. China E-mail: hanzhongchao@health-biotech.com

Professor Miao Chen, Department of Intensive Care Unit, Affiliated Hospital of Zunyi Medical University, 149 Dalian Road, Zunyi, Guizhou 563003, P.R. China

E-mail: 764590955@qq.com

*Contributed equally

Abbreviations: hUC-MSCs, human umbilical cord-derived mesenchymal stem/stromal cells; SARS-CoV-2, severe acute respiratory syndrome coronavirus 2; COVID-19, coronavirus disease 2019; ARDS, acute respiratory distress syndrome; NHC, National Health Commission; ACE2, angiotensin I converting enzyme 2 receptor; MERS-CoV, Middle East respiratory syndrome coronavirus; APACHE II, acute physiology and chronic health evaluation II; SOFA, sequential organ failure assessment; PSI, pneumonia severity index; MODS, multiple organ dysfunction syndrome; WBCs, white blood cells; OT, oxygen treatment; OI, oxygenation index; BNP, brain natriuretic peptide; TNT, troponin T; SCR, serum creatinine; P, pulse; R, respiration; BUN, blood urea nitrogen; $\mathrm{SpO}_{2}$, oxygen saturation; $\mathrm{FiO}_{2}$, fraction of inspired $\mathrm{O}_{2}$; ICU, intensive care unit; NIV, non-invasive ventilator-assisted breathing; RT-qPCR, reverse transcription-quantitative polymerase chain reaction

Key words: hUC-MSCs, COVID-19, SARS-CoV-2 coronavirus 2 (SARS-CoV-2) has spread worldwide and has become a public health emergency, principally due to the severe inflammatory response and cytokine storm, as well as the deficiency of specific vaccines and antiviral drugs. Despite the fact that mesenchymal stem cells (MSCs) are acknowledged to possess unique immunomodulatory capabilities and have promising prospects in regenerative medicine, the efficacy of the use of MSCs on critically ill elderly patients with COVID-19 is largely obscure. In the present study, a 75-year-old male patient critically ill with COVID-19 was enrolled with multiple concomitant diseases, including hypertension (for $>10$ years), diabetes and coronary heart disease (for $>1$ year). Systemic infusions of $5 \times 10^{7}$ human umbilical cord-derived mesenchymal stem/stromal cells (hUC-MSCs) were accomplished through intravenous injection every 3 days for 3 times following the consents of the patient and the relevant ethics committee. The clinical and functional outcomes, including general symptoms, vital signs, hemogram, chest radiography and the disease score, together with inflammatory factors and SARS-CoV-2 nucleic acid detection, were observed and dynamically recorded for 1 month. With the aid of hUC-MSC administration, the severe COVID-19-associated symptoms of the elderly patient, and in particular, pneumonia and the cytokine storm were continuously ameliorated. Moreover, the outcomes of multiple concomitant diseases were improved, whereas no deterioration and secondary infections, such as fever and allergic reaction were observed. The present study thus indicated that hUC-MSC administration can ameliorate the outcomes of patients with COVID-19. This type of treatment may prove to be an ideal alternative option for the treatment of patients with COVID-19, even for critically ill elderly patients with multisystemic complications. 


\section{Introduction}

Since the first report of pneumonia due to infection with the novel severe acute respiratory syndrome coronavirus 2 (SARS-CoV-2) in Wuhan, China, namely infection with coronavirus disease 2019 (COVID-19), the ongoing outbreak of the virus has rapidly spread worldwide and has become a public health emergency (1-3). To date, $>26.7$ million individuals in $>100$ hundred countries and regions were identified to have been infected with SARS-CoV-2, according to the COVID-19 dashboard by Johns Hopkins University. Of these, elderly patients are inclined to suffer from more severe symptoms and a higher intensive care unit (ICU) admission rate than other subpopulations (4,5). Generally, patients with COVID-19 infection exhibit clinical features, including fever and sputum, paroxysmal cough, gastrorrhagia, pneumothorax, shortness of breath, a low oxygen saturation, ground-glass opacity, pneumonia infiltration and in particular, critically ill patients exhibit what is known as the cytokine storm, the dysregulation of immunocytes and multiple concomitant symptoms commonly resulting in worse outcomes and a higher mortality rate $(3,5,6)$.

State-of-the-art updates on clinical grade vaccines and antiviral drugs for COVID-19 treatment are promising, but are not yet forthcoming (7-9). For instance, recent studies have revealed the involvement and ultrastructure of angiotensin I converting enzyme 2 receptor (ACE2) in mediating SARS-CoV-2 infection, which has been demonstrated as the primary cell attachment molecule for SARS-CoV-2, and holds promise for exploring the pathogenesis and drug development (9-14). Moreover, comprehensive treatments have been developed in clinical trials to prevent and reverse the cytokine storm and pulmonary injury caused by SARS-CoV-2, including spectrum antibiotics, antiviral drugs, anti-inflammatory corticosteroids, Chinese medicine, cytotherapy, immunotherapeutics and supportive therapeutics $(7,9,14-18)$. However, as for elderly patients critically ill with COVID-19, particularly those with multiple concomitant diseases, the efficacy is far from satisfactory due to the hypoimmunity and multiple organ failure, as well as the potentially controversial and severe side-effects; thus, the development of safe and effective treatment strategies if a matter of urgency $(7,18,19)$.

Mesenchymal stem/stromal cells (MSCs) possess advantaged immunomodulatory and hematopoietic-supporting properties, together with a multi-lineage differentiation potential; they have been identified from numerous tissues and hold great promise for use in regenerative medicine (20-23). Generally, the therapeutic effects of MSCs, include immunoregulation and tissue repair, mainly through direct- or trans-differentiation, in an autocrine and paracrine manner, as well as by supplying the microenvironment and homing methods (24-28). For decades, low-immunogenic MSCs have been shown to be safe and preferable for use in the management of recurrent and refractory diseases, such as spinal injury, type 2 diabetes, graft-vs.-host disease (GVHD), autoimmune diseases and hematological malignancies, as well as for severe influenza H5N1 caused by acute respiratory distress syndrome (ARDS) (20,24,29-31). Recently, Leng et al and Wang et al reported the exploration of adult tissue-derived MSCs upon 7 mild and severe patients and revealed a favorable prognosis $(14,32)$. According to the ClinicalTrials.gov of NIH, a total number of 10 clinical trials focusing on MSC-based
COVID-19 treatment from China, France, Brazil and Jordan (https:/clinicaltrials.gov/ct2/results?cond=Covid19\&term=me senchymal+stem+cells \& cntry $=\&$ state $=\&$ city $=\&$ dist $=)$ have been newly registered among the 1,074 clinical trials upon MSCs (https://clinicaltrials.gov/ct2/results?cond=\&term=mes enchymal+stem+cells $\&$ cntry $=\&$ state $=\&$ city $=\&$ dist $=$ ), which indicate the expectations of clinicians upon MSC-based cytotherapy for ameliorating the outcomes of patients with COVID-19; however, the unequivocal clinical efficacy remains to be determined.

In the present study, an elderly patient critically ill with COVID-19 with multiple concomitant diseases was enrolled, and a systemic human umbilical cord-derived mesenchymal stem/stromal cell (hUC-MSC) transplantation pilot study was conducted following the informed consent of the patient and the Ethics Committee of the Affiliated Hospital of Zunyi Medical University. Following the administration of hUC-MSCs for 3 times, the clinical symptoms and physical assessments were effectively ameliorated and non-adverse effects were observed during cytotherapy.

\section{Case report}

General information of the patient with COVID-19. The enrolled 75-year-old male patient (body weight, $50 \mathrm{~kg}$ ), with multiple comorbidities, including hypertension (for $>10$ years), diabetes (for $>1$ year), and coronary heart disease with auricular fibrillation (for $>1$ year), was first diagnosed at the local county people's hospital on January 29, 2020 due to the feeling of limb weakness for 3 days (Table SI). As described, despite without the history of offsite residence or exotic traveling, he had a history of contact with individuals from the infective spot. On January 31, 2020, the patient was in a critical condition and was transferred to the Affiliated Hospital of Zunyi Medical University for further diagnosis and treatment (Fig. 1 and Table SI).

The patient was confirmed to have been infected with COVID-19 based on the positive results of SARS-CoV-2 nucleic acid analysis in the lixivium of nasopharyngeal swab, together with the typical and multiple ground-glass opacity of exudative lesions in both lungs (Fig. 2A and Data S1). According to the systemic examination, the patient exhibited the following physical signs: A low fever $\left(37.6^{\circ} \mathrm{C}\right)$; pulse $(\mathrm{P})$, 54 beats per minute (bpm); respiration (R), $45 \mathrm{bpm}$; blood pressure (BP), 136/79 mmHg; oxygen saturation $\left(\mathrm{SpO}_{2}\right)$, 95-98\%; non-invasive ventilator-assisted breathing (NIV); fraction of inspired $\mathrm{O}_{2}\left(\mathrm{FiO}_{2}\right), 70-80 \%$; oxygenation index (OI), $92.95 \mathrm{mmHg}$ (Table SI). The patient was conscious and listless, exhibited an acute appearance and emaciated body, together with arrhythmia and atrial fibrillation (Fig. 1A). Although there were no abnormalities, such as cyanosis, tracheal deviation and symmetrical thorax, thick breathing sounds, as well as moist rales could be heard in both lungs. With the aid of a blood routine detector for auxiliary examination, it was found that the patient had a decreased content of white blood cells (WBCs; 3.76×10\%/1) and lymphocytes (LYMPH, 0.03\%) (Table SI).

Traditionally comprehensive treatment not effective for the patient. On the basis of the abovementioned information, the hospital specialist group came to the following conclusion: The patient was a critically ill with COVID-19 with severe 
A Symptoms of the critically ill COVID-19 patients according to day of illness, Jan. 31 Apr. 16, 2020

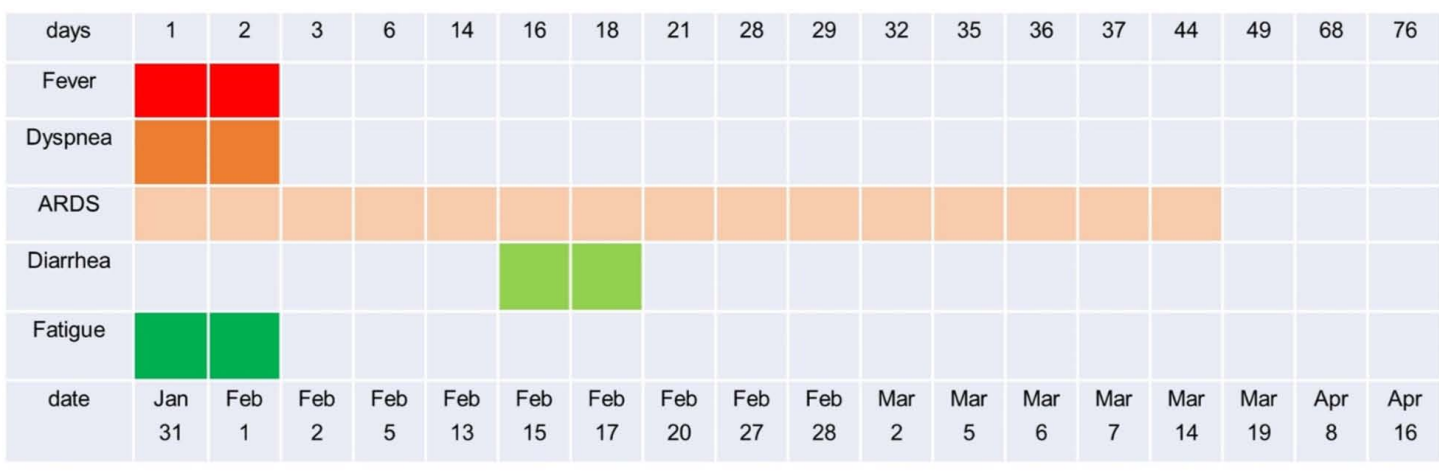

B Treatment of the critically ill COVID-19 patients according to day of illness, Jan. 31 Apr. 16, 2020

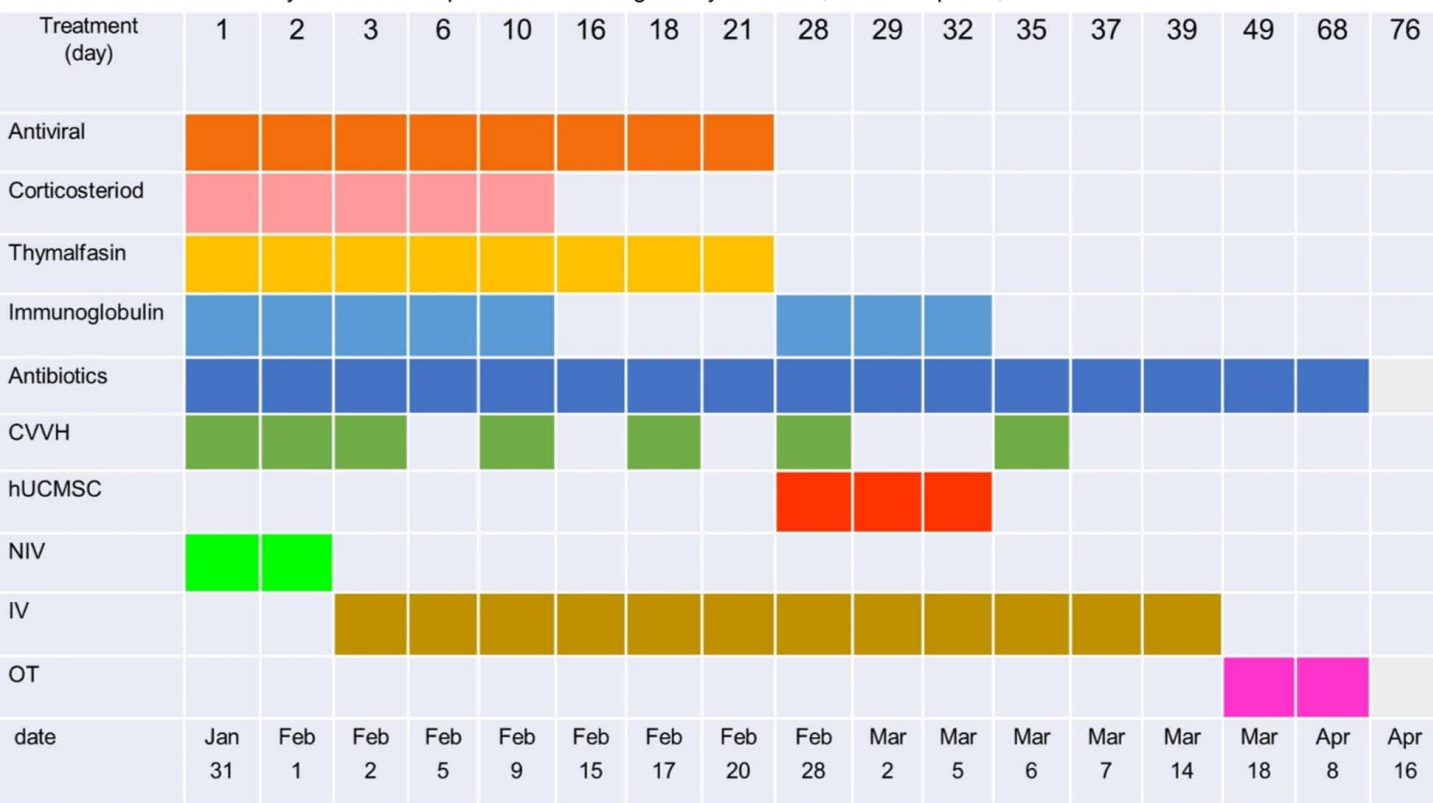

Figure 1. The dynamic symptoms and treatment of the patient critically ill with COVID-19. (A) The dynamic symptoms of the patient critically ill with COVID-19 according to the day of illness, from January 31 to April 16, 2020. (B) Treatment of the critically ill COVID-19 patient according to day of illness, January 31 to April 16, 2020.

ARDS; the patient also suffered from multiple organ dysfunction syndrome (MODS) for respiratory, kidney and liver function; coronary atherosclerotic heart disease with auricular fibrillation; and type 2 diabetes. Thereafter, the critically ill patient with COVID-19 with comorbidities was administered to the the intensive care unit (ICU) for monitoring electrocardiogram (ECG), blood oxygen saturation and dynamic blood pressure and NIV. Moreover, according to the guidance of National Health Commission (NHC) of China, comprehensive treatment was conducted on the patient, including anti-viral and anti-infection, immune enhancement, hypoglycemic management and nutrition therapy, as well as pharmacotherapy, such as lopinavir/ritonavir, ribavirin, recombinant human interferon $\alpha 2 \mathrm{~b}$ (hIL- $\alpha 2 \mathrm{~b})$ and abidor (Fig. 1B). On February 2, 2020, the patient was administered tracheal intubation followed by tracheotomy (the following day) for invasive breathing (IV) supportive therapy, and numerous pink frothy sputa were visible. However, even though following a 30-day period of positive support care and symptomatic therapy, the enrolled patient was not able to be removed ventilator support due to the poor absorption of infiltration shadow in the lungs (Figs. 1B, and 2B and C, and Table SI).
hUC-MSC administration and safety outcomes. Considering the persistent poor prognosis of rehabilitative treatment and the shortfall of substantial improvement of the patient, the hospital specialist group unanimously gave the consultation for the application for MSC-based cytotherapy, which was subsequently approved by the patient and by the Ethics Committee of the Affiliated Hospital of Zunyi Medical University (Figs. 1, 2C and D).

Having taken into sufficient consideration the potential untoward effects and following the meticulous arrangement of emergency measures, the patient was subjected to the intravenous delivery of clinical-grade hUC-MSCs on February 28, March 2 and March 5, respectively (Fig. 1B). In total, $5 \times 10^{7}$ hUC-MSCs (Jiangxi Health-Biotech Stem Cell Technology Co. Ltd, product lot no. 202002JF07, 202003JF02 and 202002JF05) in $100 \mathrm{ml}$ saline were infused into the body of the patient through a blood transfusion needle at a certain rate of 40 drops per min. Within $24 \mathrm{~h}$ after the transfusion process, no acute untoward effects, such as infusion-associated and allergic reactions (e.g., instantaneous low fever, polypnea or slight trembling) were observed in the patient. Furthermore, 

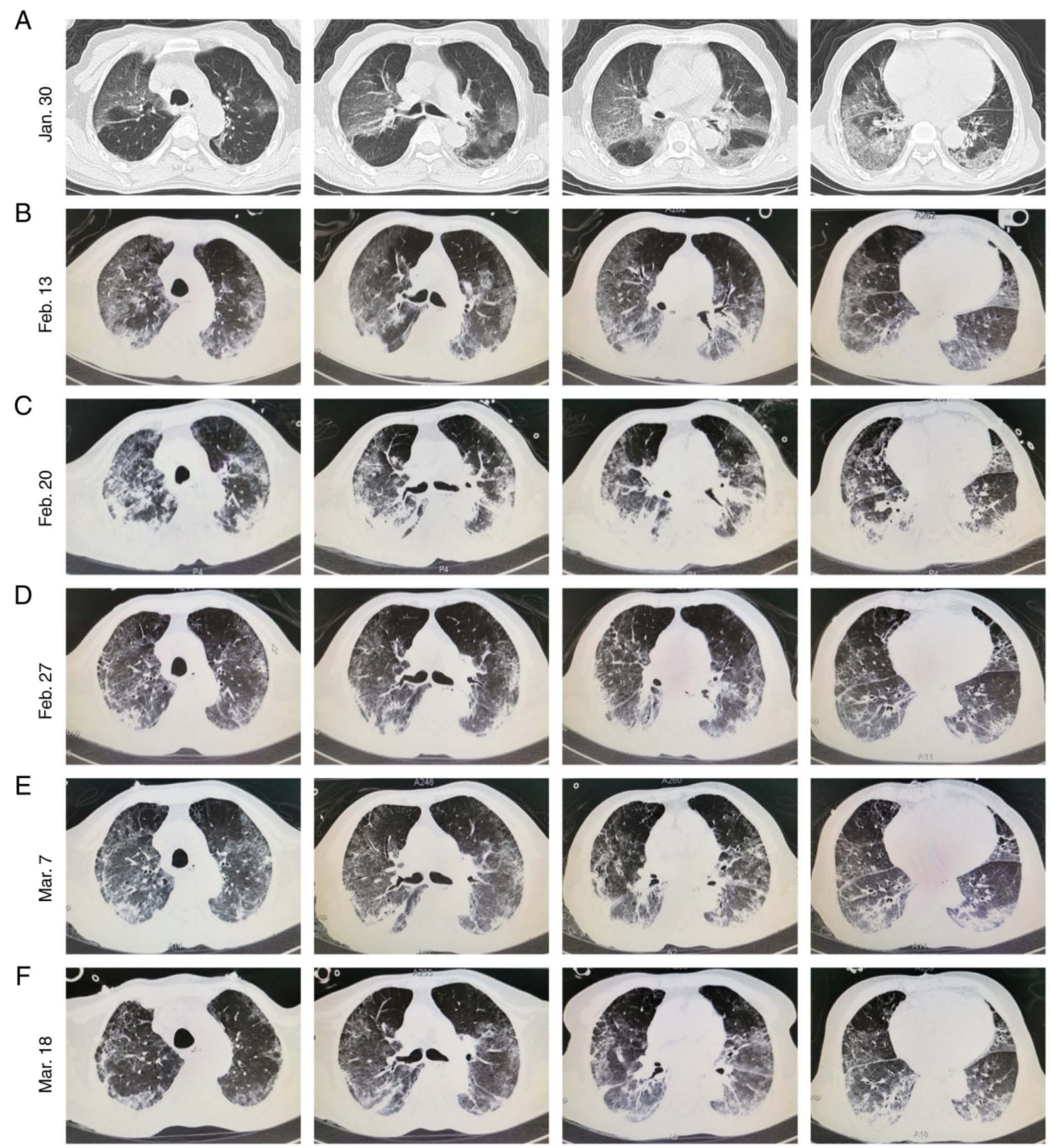

G
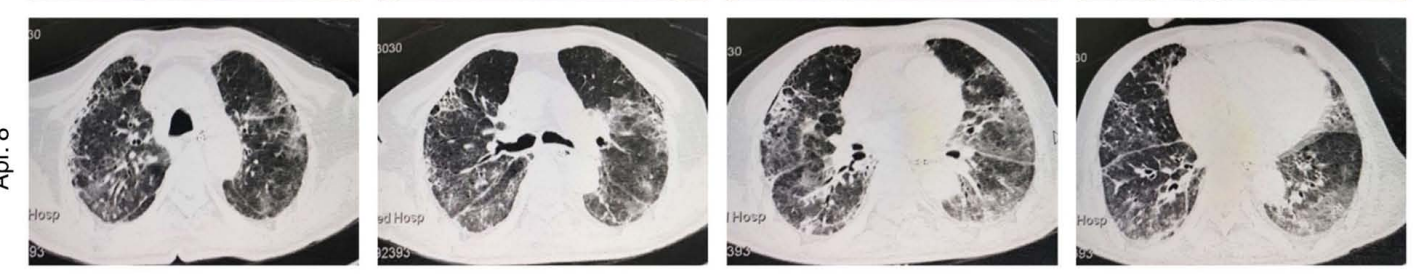

Figure 2. The dynamic chest radiographs during traditionally comprehensive treatment and hUC-MSC administration. (A-C) The dynamic chest X-ray images of the patient on (A) January 30, (B) February 13 and (C) February 20,2020. The streaky ground-glass opacity of exudative lesions in both lungs was steadily increased in density over time during traditionally comprehensive treatment. (D-G) The dynamic chest X-ray images of the patient on February 27 (D, the day before the first trial for hUC-MSC transplantation), March 7 (E, 2 days after the third trial for hUC-MSC transplantation), March 18 (F, 13 days after the third trial for hUC-MSC transplantation), and April 8 (G, 34 days after the third trial for hUC-MSC transplantation), 2020. The streaky ground-glass opacity of exudative lesions in both lungs was steadily decreased in density over time during hUC-MSC administration with traditionally comprehensive treatment. hUC-MSC, human umbilical cord-derived mesenchymal stem/stromal cell.

no secondary infection, delayed hypersensitivity or life-threatening events occurred in the following 30 days of observation. Furthermore, accompanied by the upregulation of immunoglobulin, the clinical parameters, chest radiography and a SARS-CoV-2 nucleic acid test, as well as the aforementioned comprehensive treatment, including antibiotics, antiviral drugs and respiratory support by the breathing machine, were recorded (Fig. 2E and F). The symptoms observed prior to hUC-MSC administration, such as fatigue and listlessness, low oxygen saturation and an acute appearance disappeared, and arrhythmia and atrial fibrillation in the patient were improved to a certain extent as well (Table SI). 
A

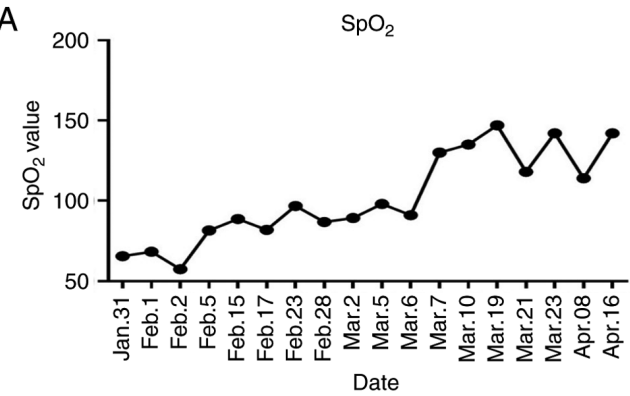

C

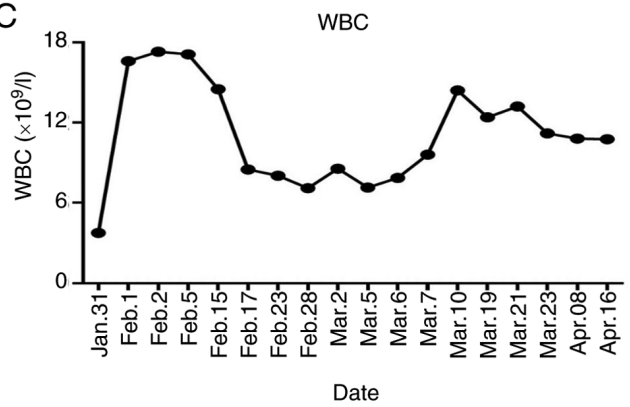

E

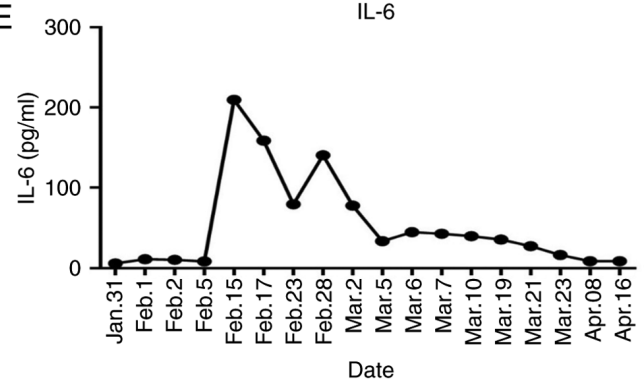

G

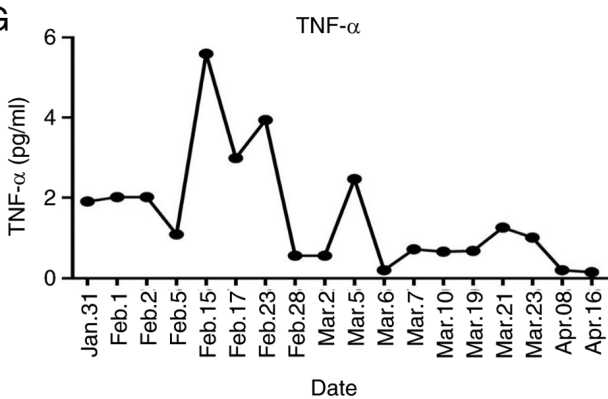

B

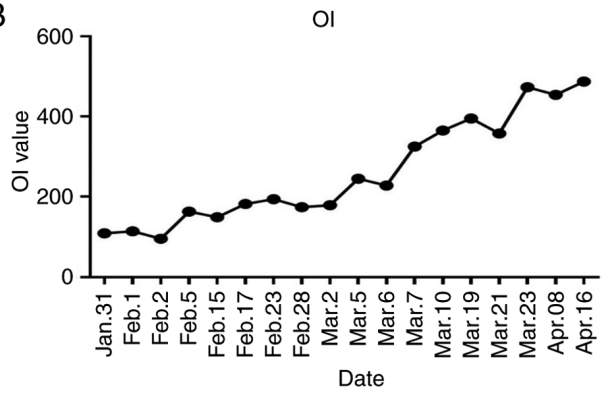

D

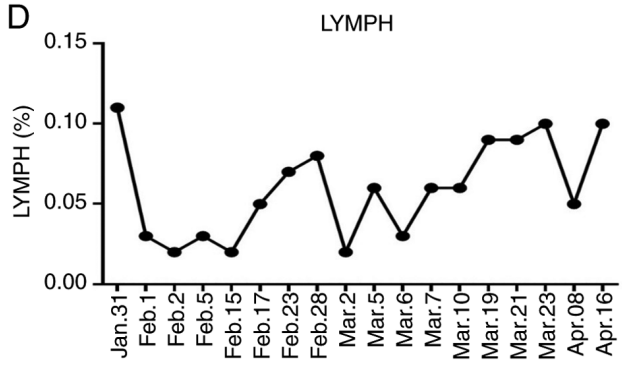

Date
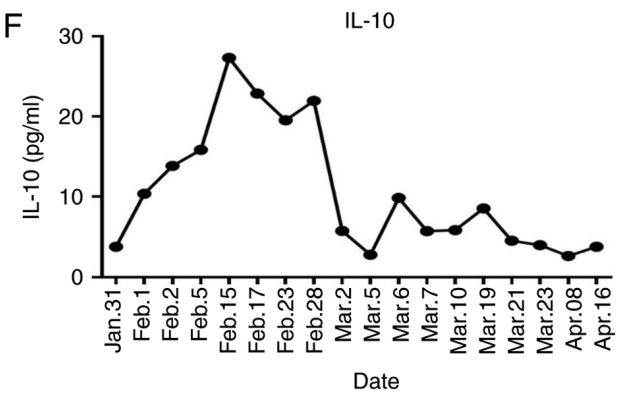

$\mathrm{H}^{6} \quad 6 \quad{ }^{\text {TNF- }-\gamma}$

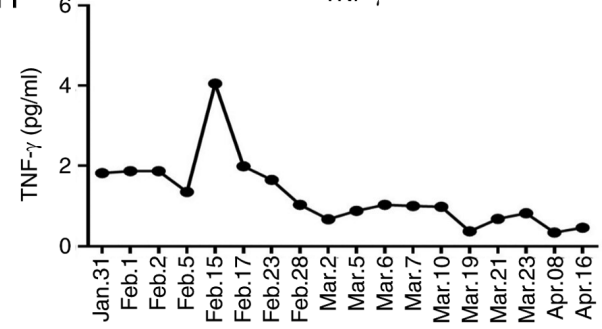

Date

Figure 3. The dynamic variations of clinical parameters during the patient treatment. (A and B) The dynamic upregulation of (A) $\mathrm{SpO}_{2}$ value and (B) $\mathrm{OI}$ value in the patient from Janruary 31 to April 16, 2020. (C and D) The dynamic upregulation of WBCs (C, 10\%/l) and LYMPH (D, \%) in the peripheral blood of the patient from January 31 to April 16, 2020. (E-H) The dynamic immunosuppression of IL-6 (E, pg/ml), IL-10 (F, pg/ml), TNF- $\alpha$ (G, pg/ml), TNF- $\gamma$ (H, pg/ml) in the peripheral blood of the patient from January 31 to April 16th, 2020. SpO2, oxygen saturation; OI, oxygenation index; WBCs, white blood cells; LYMPH, lymphocytes.

hUC-MSC administration ameliorates the outcomes of COVID-19-associated pneumonia. To systematically and meticulously dissect the efficacy of systemic hUC-MSC transplantation upon the patient critically ill with COVID-19 with multiple comorbidities, the chest radiography was primarily used. Compared with the CT image of the chest on February 27 (the day before MSC treatment), the first application of hUC-MSCs (within 2 days since hUC-MSC transplantation) did not reveal immediate and evidently ameliorative effects upon the absorption of infiltration shadow in the lungs, even though a series of the aforementioned vital signs were significantly improved (Fig. 2D and E). However, 13 days after the third trial of hUC-MSC transplantation, pneumonia infiltration was suppressed, and in particular, spontaneous remission in the ground-glass opacity was observed in both lungs on April 8 (Fig. 2F and G).

hUC-MSC administration ameliorates the clinical outcomes of the patient. From the view of the respiratory support curve, it was found that both the $\mathrm{SpO}_{2}$ and OI were upregulated, whereas the $\mathrm{FiO}_{2}$ was decreased, which was further confirmed by the replacement of mechanical ventilation with ordinary oxygen treatment (OT) (Figs. 1B, and $3 \mathrm{~A}$ and B, and Table SI). Together with the hemoglobin $(\mathrm{Hb})$ and platelet (PLT) values, a clinical laboratory examination revealed the ultimate resumption of the content of WBCs and total lymphocyte count in the peripheral blood of patient after 2 weeks from the hUC-MSC transplantation (Figs. 3C and D, and S1 A and B, and Table SI). 
A

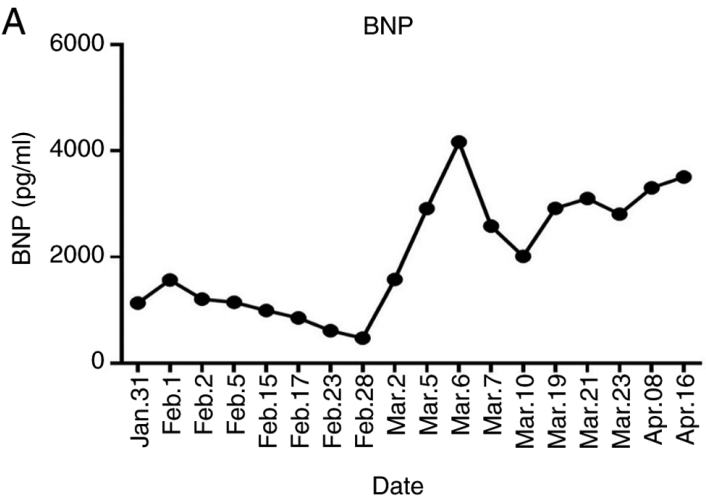

C

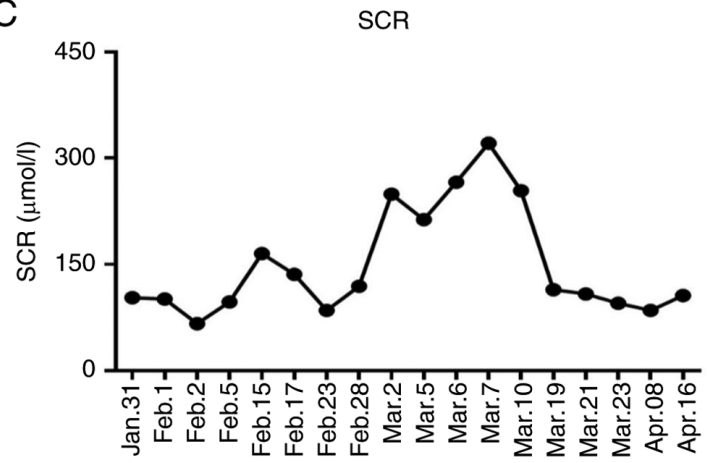

Date

E

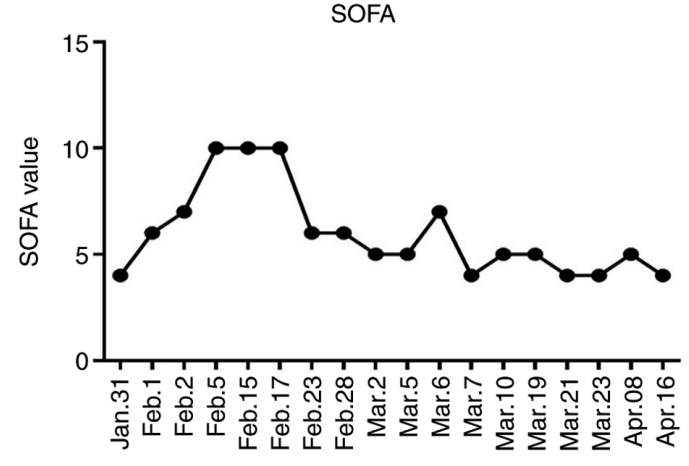

B

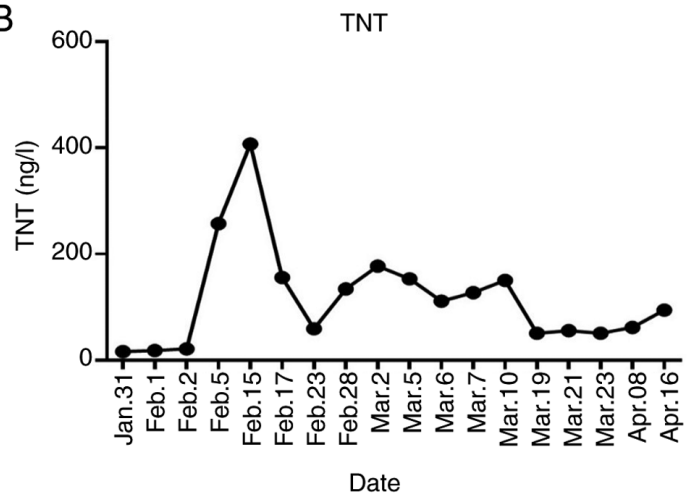

D

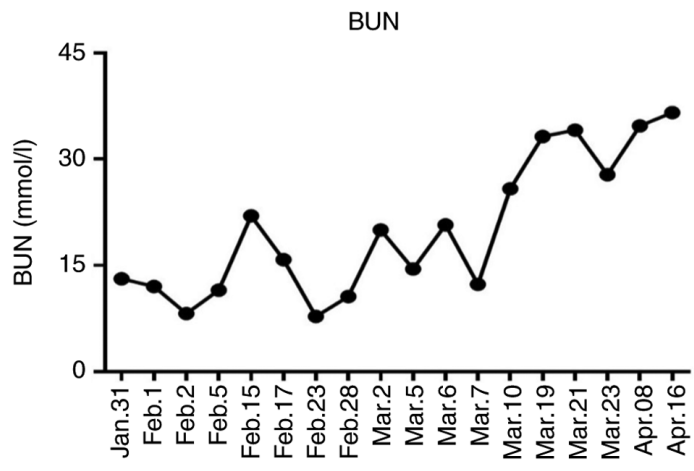

Date

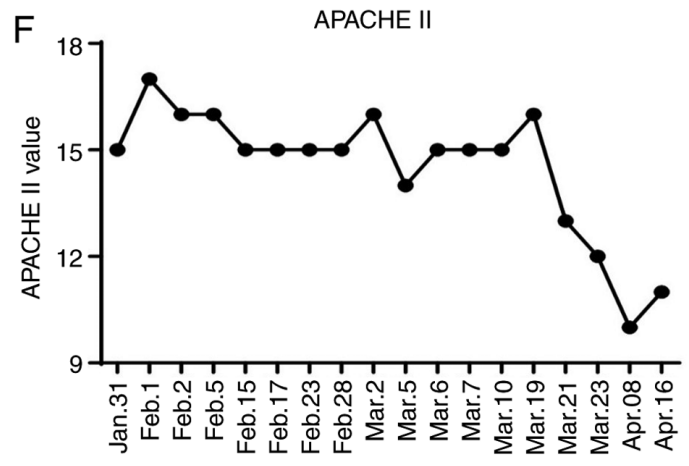

Date

Figure 4. The dynamic variations of multiple comorbidities-associated parameters and the severity of illness during the patient treatment. (A and B) The dynamic variation of heart-related indicators including the upregulation of BNP $(\mathrm{A}, \mathrm{pg} / \mathrm{ml})$ and downregulation of $\mathrm{TNT}(\mathrm{B}, \mathrm{ng} / \mathrm{l})$ in the patient from January 31 to March 23th, 2020. (C and D) The dynamic variation of kidney-related indicators including the instantaneous upregulation of SCR (C, $\mu$ mol/l) and delayed upregulation of BUN (D, mmol/l) in the patient from January 31 to April 16, 2020. (E and F) The dynamic variation of severity of illness-associated indicators including the standard (E) SOFA value, and (F) APACHE II value in the patient from January 31 to April 16, 2020. BNP, brain natriuretic peptide; TNT, troponin T; BUN, blood urea nitrogen; APACHE II, acute physiology and chronic health evaluation II; SOFA, sequential organ failure assessment.

Furthermore, to assess the potential effects on the cytokine storm, a sharp decrease was observed in the levels of pivotal pro-inflammatory factors, including IL-2, IL-4, IL-6, IL-10, TNF- $\alpha$ and TNF- $\gamma$ in the patient critically ill with COVID-19 (Figs. 3E-H, and S1C and D, and Table SI).

Simultaneously, the expression levels of heart-associated [brain natriuretic peptide (BNP), Troponin T (TNT)], kidney-associated [serum creatinine (SCR), blood urea nitrogen (BUN)] and liver-associated [alanine transaminase (ALT), aspartate aminotransferase (AST), albumin (ALB) and pre-serum protein $\mathrm{PA})]$ biomarkers were partially recovered following a transient increase (Figs. 4A-D, and S2A-D, and Table SI). Collectively, these results indicated the ameliorative outcomes of MODS and multiple comorbidities (e.g., coronary atherosclerotic heart disease with auricular fibrillation).

For the purpose of comprehensively evaluating the efficacy of hUC-MSCs on COVID-19 infection, the multifaceted disease scores were conducted according to the standard indexes. From the dynamic variations of the curves, it was found tht both the sequential organ failure assessment (SOFA) and pneumonia severity index (PSI) scores were immediately decreased after the third trial of hUC-MSC transplantation, while the Acute physiology and chronic health evaluation II (PACHE II) exhibited a delayed decrease for 2 weeks (Figs. 4E and F, and S2E). Above all, with the aid of hUC-MSC administration and traditionally comprehensive treatment, 
the SARS-CoV-2 nucleic acid test result of the patient finally yielded a negative result as well.

\section{Discussion}

The ongoing outbreak of COVID-19 pneumonia caused by SARS-CoV-2 has become a worldwide public hygiene event and has caused the death of $>877,500$ individuals due to a severe inflammation response and cytokine storm, particularly for critically ill elderly patients with pneumonia-induced ARDS and multiple comorbidities (3-6,33). Due to the deficiency of targeted therapeutics, as well as specialized antiviral vaccines and drugs, there is an urgency for the development of MSC-based cytotherapy to reverse the immunodysregulation and high mortality, and to facilitate functional reconstruction $(8,9,18)$. The present study is a pilot case report study on the systemic infusion of hUC-MSCs for patients critically ill with COVID-19. On the basis of traditionally comprehensive treatment, hUC-MSC administration did not trigger primary or secondary untoward effects, but effectively ameliorated the outcomes of the patient, such as vital signs, deteriorated clinical parameters and pneumonia associated with SARS-CoV-2 infection, and related ARDS and MODS.

In general,SARS-CoV-2 belongs to the genus and exhibits great phylogenetic similarity to the coronavirus from bats, which is distinguished from the 2003 SARS-CoV and the Middle East respiratory syndrome coronavirus (MERS-CoV) at the molecular level (7,8,32,34-37). Despite the early outbreak of COVID-19 in Wuhan of China, yet increasingly evidences were inclined to dismiss the possibility from the classification of SARS-CoV-2 $(33,34,37,38)$. Although patient zero and the spectrum of intermediaries are still unknowable, yet the multifaceted characterization of latent period and asymptomatic carriers together with the main routes of human-to-human transmission and comprehensive diagnosis have made the prevention and control of COVID-19 outbreaks in the early period of exponential growth more available $(3,7,37,39)$. For instance, the suspected SARS-CoV-2-infected patients in China are diagnosed and quarantined on the basis of evidence, including epidemiological, demographic, clinical, laboratory and radiological characteristics according to guidance for the diagnosis and treatment of 2019 novel coronavirus infected pneumonia from China CDC and WHO recommendations $(4-6,15)$. For instance, numerous strategies have been developed for SARS-CoV-2 detection, including SARS-CoV-2 RNA and protein, and even the early ELISA- or collaurum-based antibody response ( $\operatorname{IgG}, \operatorname{IgM}$ and $\operatorname{IgA}$ ) against the virus (9). Although traditionally comprehensive treatment, including supportive symptomatic treatment (e.g., steroids, oxygen therapy and fluid management) and therapeutic intervention (e.g., immunomodulatory adjuvants and substances) plays a critical role in the treatment of COVID-19-infected patients, the efficacy of suppressing transmission and ameliorating disease is far from satisfactory, due to the time-consuming development for unequivocal clinical-grade targeted vaccines and antiviral drugs $(9,19,40)$. For example, scientific or clinical data upon broad-spectrum antiviral drug repurposing, high-throughput drug screening, late humoral response and persistence of alternative neutralizing antibodies during SARS-CoV-2 infection is desperately scarce (9). Worse still, the controversial outcome and the side-effects of therapeutic drugs, such as gastrorrhagia and osteoporosis are suspected to be caused by glucocorticoids $(18,19)$. Moreover, elderly patients are apt to suffer from worse symptoms (e.g., ARDS), and are associated with a higher ICU admission rate and higher mortality rate when compared with other demographics $(6,33)$. To date, basic research and clinical trials have indicated the pivotal role of higher concentrations of pro-inflammatory factors (e.g., IL-6, IL-10, G-CSF, MCP-1 and TNF- $\alpha$ ) and the resultant severe cytokine storm, which further accelerate the ground-glass opacity and pneumonia infiltration, as well as multiple organ destruction and dysfunction in the critically ill COVID-19 patient $(3,5)$. Above all, despite the large flow of scientific or clinical explorations on mechanistic characteristics and possible drug repositioning rationale, the eventual mitigation and containment of this COVID-19 epidemic diseases still depends on the current exponentially increasing knowledge of the SARS-CoV-2 pathology, virology and cross-species transmission (9).

Since the development of new drugs is a time-consuming and costly process, drug repurposing, such as MSCs- and the gene therapy-based approach is a promising strategy for identifying therapeutic solutions during the time-critical pandemic $(9,41)$. Since the 1960 s, MSCs were initially isolated from bone marrow followed by various adult tissues (e.g., adipose tissue and dental pulp) and perinatal tissues (e.g., umbilical cord and placenta), as well as human pluripotent stem cells (e.g., hESCs, iPSCs) (20,42-44). Of the aforementioned MSCs, hBM-MSCs and hUC-MSCs are associated with the most applications and long-term in vitro proliferation capacity, respectively $(20,24)$. For decades, the authors of the present study, as well as others, have reported the clinical and preclinical applicability of MSCs in refractory and recurrent diseases, such as Crohn's disease, aplastic anemia, acute myocardial infarction, GVHD and even H5N1-infected pneumonia which cause acute lung injury and acute respiratory distress syndrome (ALI/ARDS), which are similar to the symptoms of SARS-CoV-2 infection $(20,24,25,42,43,45)$. Very recently, Leng et al and Wang et al reported the clinical remission of a 65-year-old female critically ill patient with COVID-19 with hUC-MSC transplantation for 3 times as well, whereas the lung damage was relatively mild, which was confirmed by another studies $(14,32)$. However, the safety and effectiveness assessment on elderly patients critically ill with COVID-19 with severe pneumonia-induced ARDS and MODS-associated multiple comorbidities remains largely unknown. Herein, prior to potentially large-scale clinical investigations and applications, the feasibility and dependability of hUC-MSC administration on a critically ill elderly patient with COVID were preliminarily confirmed, including immunodysfunction, cytokine storm, severe pneumonia-induced ARDS, MODS and the concomitant comorbidities as well, which add new references to MSC-based COVID-19 treatment, particularly for patients without a therapeutic response to traditional medications, such as immunoglobulins, hormones, antibiotics and anti-viral vaccines $(14,46)$. Furthermore, it was also realized that the case report alone is far from sufficient for the full interpretation of MSC-based therapy for COVID-19-infected patients. Therefore, the replicability of the results obtained with this representative patient are not sufficient. Additionally, the potentially bidirectional immunoregulation effects and equilibrium upon cytokine storm inhibition and SARS-CoV-2 
replication should not be neglected, and the safety and effectiveness assessment of MSC-based cytotherapy on COVID-19-infected patients should be further examined in clinical trials with a large number of patients.

\section{Acknowledgements}

Not applicable.

\section{Funding}

The present study was supported by the National Natural Science Foundation of China (grant nos. 81960024, 81700119 and 81900126), the Major Program of the National Natural Science Foundation of China (grant no. 81330015), the National Science and Technology Major Projects of China for 'Major New Drugs Innovation and Development' (grant no. 2014ZX09508002-003), the Natural Science Foundation of Tianjin (grant no. 19JCQNJC12500), the Project funded by China Postdoctoral Science Foundation (grant no. 2019M661033), the Natural Science Foundation of Hebei (grant no. H2020206403), the Emergency Project funded by Department of Science and Technology of Jiangxi Province (2020, to ZHa), the Key project funded by Department of Science and Technology of Shangrao City (2020, to ZHa) and the Science and Technology Project of Tianjin (grant no. 17ZXSCSY00030).

\section{Availability of data and materials}

The data used to support the findings of the study are included in the present article. Additional data related to this study are also available from the corresponding author. Trial registration: The safety and effectiveness of human umbilical cord mesenchymal stem cells in the treatment of acute respiratory distress syndrome of severe novel coronavirus pneumonia (COVID-19), ChiCTR2000030116, was registered on February 23, 2020 (http://www.chictr.org.cn/showproj. aspx?proj=49901; prospectively registered). The analysis of clinical characteristics and therapeutic effect of 9 cases of novel coronavirus pneumonia (COVID-19), ChiCTR2000031930 was registered on April 15, 2020 (http://www.chictr.org. $\mathrm{cn} /$ showproj.aspx?proj=51602; retrospective registration).

\section{Authors' contributions}

$\mathrm{HC}, \mathrm{LZ}$ and ZHe were involved in the collection and assembly of data and manuscript writing. DW, LL, WZ and TC were involved in the collection and assembly of data. ZD was involved in the preparation of the hUC-MSCs. LZ and $\mathrm{ZHa}$ were involved in data interpretation, manuscript writing and revision. $\mathrm{LZ}, \mathrm{MC}$ and $\mathrm{ZHa}$ were involved in the conception and design of the study, and in data analysis. All authors read and approved the final manuscript.

\section{Ethics approval and consent to participate}

The procedures performed on the patient followed the internationally recognized guidelines. The ethical approval of the research was signed by the Ethics Committee of the
Affiliated Hospital of Zunyi Medical University in China (approval no. KLL-2020-013).

\section{Patient consent for publication}

The patient signed an informed consent to the publication of his case report.

\section{Competing interests}

The authors declare no that they have no competing interests.

\section{References}

1. Holshue ML, DeBolt C, Lindquist S, Lofy KH, Wiesman J, Bruce $\mathrm{H}$, Spitters $\mathrm{C}$, Ericson $\mathrm{K}$, Wilkerson $\mathrm{S}$, Tural A, et al: First case of 2019 novel coronavirus in the United States. N Engl J Med 382: 929-936, 2020.

2. Li JY, You Z, Wang Q, Zhou ZJ, Qiu Y, Luo R and Ge XY: The epidemic of 2019-novel-coronavirus (2019-nCoV) pneumonia and insights for emerging infectious diseases in the future. Microbes Infect 22: 80-85, 2020

3. Huang C, Wang Y, Li X, Ren L, Zhao J, Hu Y, Zhang L, Fan G, Xu J, Gu X, et al: Clinical features of patients infected with 2019 novel coronavirus in Wuhan, China. Lancet 395: 497-506, 2020.

4. Li Q, Guan X, Wu P, Wang X, Zhou L, Tong Y, Ren R, Leung KSM, Lau EHY, Wong JY, et al: Early transmission dynamics in wuhan, China, of novel coronavirus-infected pneumonia. N Engl J Med 382: 1199-1207, 2020.

5. Chen N, Zhou M, Dong X, Qu J, Gong F, Han Y, Qiu Y, Wang J, Liu Y, Wei Y, et al: Epidemiological and Clinical characteristics of 99 cases of 2019 novel coronavirus pneumonia in Wuhan, China: A descriptive study. Lancet 395: 507-513, 2020.

6. Wang D, Hu B, Hu C, Zhu F, Liu X, Zhang J, Wang B, Xiang H, Cheng Z, Xiong Y, et al: Clinical characteristics of 138 hospitalized patients with 2019 novel Coronavirus-infected pneumonia in Wuhan, China. JAMA 323: 1061-1069, 2020.

7. Jiang S, Du L and Shi Z: An emerging coronavirus causing pneumonia outbreak in Wuhan, China: Calling for developing therapeutic and prophylactic strategies. Emerg Microbes Infect 9: 275-277, 2020.

8. Zhou Y, Hou Y, Shen J, Huang Y, Martin W and Cheng F: Network-based drug repurposing for novel coronavirus 2019-nCoV/SARS-CoV-2. Cell Discov 6: 14, 2020.

9. Nitulescu GM, Paunescu H, Moschos SA, Petrakis D, Nitulescu G, Ion GND, Spandidos DA, Nikolouzakis TK, Drakoulis N and Tsatsakis A: Comprehensive analysis of drugs to treat SARSCoV2 infection: Mechanistic insights into current COVID19 therapies (Review). Int J Mol Med 46: 467-488, 2020.

10. Wan Y, Shang J, Graham R, Baric RS and Li F: Receptor recognition by the novel coronavirus from Wuhan: An analysis Based on Decade-long structural studies of SARS coronavirus. J Virol 94: e00127-20: 2020.

11. Shang J, Ye G, Shi K, Wan Y, Luo C, Aihara H, Geng Q, Auerbach A and Li F: Structural basis of receptor recognition by SARS-CoV-2. Nature 581: 221-224, 2020.

12. Lan J, Ge J, Yu J, Shan S, Zhou H, Fan S, Zhang Q, Shi X, Wang Q, Zhang L and Wang X: Structure of the SARS-CoV-2 spike Receptor-binding domain bound to the ACE2 receptor. Nature 581: 215-220, 2020.

13. Kuba K, Imai Y, Rao S, Gao H, Guo F, Guan B, Huan Y, Yang P, Zhang Y, Deng W, et al: A crucial role of angiotensin converting enzyme 2 (ACE2) in SARS coronavirus-induced lung injury. Nat Med 11: 875-879, 2005.

14. Leng Z, Zhu R, Hou W, Feng Y, Yang Y, Han Q, Shan G, Meng F, Du D, Wang S, et al: Transplantation of ACE2(-) mesenchymal stem cells improves the outcome of patients with COVID-19 pneumonia. Aging Dis 11: 216-228, 2020.

15. Yu F, Du L, Ojcius DM, Pan C and Jiang S: Measures for diagnosing and treating infections by a novel coronavirus responsible for a pneumonia outbreak originating in Wuhan, China. Microbes Infect 22: 74-79, 2020.

16. Zhou G and Zhao Q: Perspectives on therapeutic neutralizing antibodies against the Novel Coronavirus SARS-CoV-2. Int J Biol Sci 16: 1718-1723, 2020. 
17. Dhama K, Sharun K, Tiwari R, Dadar M, Malik YS, Singh KP and Chaicumpa W: COVID-19, an emerging coronavirus infection: Advances and prospects in designing and developing vaccines, immunotherapeutics, and therapeutics. Hum Vaccin Immunother 16: 1232-1238, 2020.

18. Shang L, Zhao J, Hu Y, Du R and Cao B: On the use of corticosteroids for 2019-nCoV pneumonia. Lancet 395: 683-684, 2020.

19. Russell CD, Millar JE and Baillie JK: Clinical evidence does not support corticosteroid treatment for $2019-\mathrm{nCoV}$ lung injury. Lancet 395: 473-475, 2020.

20. Zhao Q, Zhang L, Wei Y, Yu H, Zou L, Huo J, Yang H, Song B, Wei T, Wu D, et al: Systematic comparison of hUC-MSCs at various passages reveals the variations of signatures and therapeutic effect on acute Graft-versus-host disease. Stem Cell Res Ther 10: 354, 2019.

21. Yao J, Chen N, Wang X, Zhang L, Huo J, Chi Y, Li Z and Han Z: Human supernumerary Teeth-derived apical papillary stem cells possess preferable characteristics and efficacy on hepatic fibrosis in mice. Stem Cells Int 2020: 6489396, 2020

22. Lu S, Ge M, Zheng Y, Li J, Feng X, Feng S, Huang J, Feng Y, Yang D, Shi J, et al: CD106 is a novel mediator of bone marrow mesenchymal stem cells via NF- $\kappa \mathrm{B}$ in the bone marrow failure of acquired aplastic anemia. Stem Cell Res Ther 8: 178, 2017

23. Du W, Li X, Chi Y, Ma F, Li Z, Yang S, Song B, Cui J, Ma T, Li J, et al: VCAM-1+ placenta chorionic villi-derived mesenchymal stem cells display potent Pro-angiogenic activity. Stem Cell Res Ther 7: 49, 2016.

24. Huo J, Zhang L, Ren X, Li C, Li X, Dong P, Zheng X, Huang J, Shao Y, Ge M, et al: Multifaceted characterization of the signatures and efficacy of mesenchymal stem/stromal cells in acquired aplastic anemia. Stem Cell Res Ther 11: 59, 2020.

25. Samsonraj RM, Raghunath $M$, Nurcombe V, Hui JH, van Wijnen AJ and Cool SM: Concise review: Multifaceted characterization of human mesenchymal stem cells for use in regenerative medicine. Stem Cells Transl Med 6: 2173-2185, 2017.

26. Pourgholaminejad A, Aghdami N, Baharvand $\mathrm{H}$ and Moazzeni SM: The effect of pro-inflammatory cytokines on immunophenotype, differentiation capacity and immunomodulatory functions of human mesenchymal stem cells. Cytokine 85: 51-60, 2016.

27. Fan XL, Zhang Y, Li X and Fu QL: Mechanisms underlying the protective effects of mesenchymal stem cell-based therapy. Cell Mol Life Sci 77: 2771-2794, 2020

28. Kfoury Y and Scadden DT: Mesenchymal cell contributions to the stem cell niche. Cell Stem Cell 16: 239-253, 2015.

29. Darwish I, Mubareka S and Liles WC: Immunomodulatory therapy for severe influenza. Expert Rev Anti Infect Ther 9. $807-822,2011$

30. Zhang X, Yang Y, Zhang L, Lu Y, Zhang Q, Fan D, Zhang Y, Zhang Y, Ye Z and Xiong D: Mesenchymal stromal cells as vehicles of tetravalent bispecific tandab (CD3/CD19) for the treatment of B cell lymphoma combined with IDO pathway inhibitor D-1-methyl-tryptophan. J Hematol Oncol 10: 56, 2017.

31. Wei Y, Zhang L, Chi Y, Ren X, Gao Y, Song B, Li C, Han Z, Zhang L and Han Z: High-efficient generation of VCAM-1(+) mesenchymal stem cells with multidimensional superiorities in signatures and efficacy on aplastic anaemia mice. Cell Prolif 53: e12862, 2020.

32. Wang L, Liu T, Liang R, Wang G, Liu Y, Zou J, Liu N, Zhang B, Liu Y, Ding X, et al: Mesenchymal stem cells ameliorate $\beta$ cell dysfunction of human type 2 diabetic islets by reversing $\beta$ cell dedifferentiation. EBioMedicine 51: 102615, 2020.
33. Xu Z, Shi L, Wang Y, Zhang J, Huang L, Zhang C, Liu S, Zhao P, Liu H, Zhu L, et al: Pathological findings of COVID-19 associated with acute respiratory distress syndrome. Lancet Respir Med 8: 420-422, 2020.

34. Lu R, Zhao X, Li J, Niu P, Yang B, Wu H, Wang W, Song H, Huang B, Zhu N, et al: Genomic characterisation and epidemiology of 2019 novel coronavirus: implications for virus origins and receptor binding. Lancet 395: 565-574, 2020.

35. Guarner J: Three emerging coronaviruses in two decades. Am J Clin Pathol 153: 420-421, 2020.

36. Meo SA, Alhowikan AM, Al-Khlaiwi T, Meo IM, Halepoto DM, Iqbal M, Usmani AM, Hajjar W and Ahmed N: Novel coronavirus 2019-nCoV: Prevalence, biological and clinical characteristics comparison with SARS-CoV and MERS-CoV. Eur Rev Med Pharmacol Sci 24: 2012-2019, 2020.

37. Forster P, Forster L, Renfrew C and Forster M: Phylogenetic network analysis of SARS-CoV-2 genomes. Proc Natl Acad Sci USA 117: 9241-9243, 2020

38. Li X, Zai J, Zhao Q, Nie Q, Li Y, Foley BT and Chaillon A: Evolutionary history, potential intermediate animal host, and cross-species analyses of SARS-CoV-2. J Med Virol 92: 602-611, 2020.

39. Riou J and Althaus CL: Pattern of early human-to-human transmission of Wuhan 2019 novel coronavirus $(2019-\mathrm{nCoV})$, December 2019 to January 2020. Euro Surveill 25: 2000058 , 2020.

40. Colson P, Rolain JM and Raoult D: Chloroquine for the 2019 novel coronavirus SARS-CoV-2. Int J Antimicrob Agents 55: $105923,2020$.

41. Nazeam J, Mohammed EZ, Raafat M, Houssein M, Elkafoury A, Hamdy D and Jamil L: Based on principles and insights of COVID-19 epidemiology, genome sequencing, and pathogenesis: Retrospective analysis of sinigrin and Prolixin(RX) (Fluphenazine) provides Off-label drug candidates. SLAS Discov: Aug 17, 2020 (Epub ahead of print). doi: $10.1177 / 2472555220950236$.

42. Zhang L, Wang H, Liu C, Wu Q, Su P, Wu D, Guo J, Zhou W, $\mathrm{Xu}$ Y, Shi L and Zhou J: MSX2 initiates and accelerates mesenchymal stem/stromal cell specification of hPSCs by regulating TWIST1 and PRAME. Stem Cell Reports 11: 497-513, 2018.

43. Wei Y, Hou H, Zhang L, Zhao N, Li C, Huo J, Liu Y, Zhang W, Li Z, Liu D, et al: JNKi- and DAC-programmed mesenchymal stem/stromal cells from hESCs facilitate hematopoiesis and alleviate hind limb ischemia. Stem Cell Res Ther 10: 186, 2019.

44. Soontararak S, Chow L, Johnson V, Coy J, Wheat W, Regan D and Dow S: Mesenchymal stem cells (MSC) derived from induced pluripotent stem cells (iPSC) equivalent to Adipose-derived MSC in promoting intestinal healing and microbiome normalization in mouse inflammatory bowel disease model. Stem Cells Transl Med 7: 456-467, 2018.

45. Liang X, Ding Y, Zhang Y, Tse HF and Lian Q: Paracrine mechanisms of mesenchymal stem cell-based therapy: Current status and perspectives. Cell Transplant 23: 1045-1059, 2014.

46. Ye J and Gimble JM: Regulation of stem cell differentiation in adipose tissue by chronic inflammation. Clin Exp Pharmacol Physiol 38: 872-878, 2011.

This work is licensed under a Creative Common Attribution-NonCommercial-NoDerivatives 4.0 International (CC BY-NC-ND 4.0) License. 